\title{
Skyrme-Hartree-Fock approach for description of static nuclear properties of well-deformed nuclei
}

\author{
Meng-Hock Koh a, b, Nurhafiza Mohamad Nor a, Nor-Anita Rezle ${ }^{a}$, Kai-Wen Kelvin Lee ${ }^{\text {a }}$, Philippe \\ Quentin ${ }^{\mathrm{c}}$, Norehan Mohd Nor ${ }^{\mathrm{a}}$, Ludovic Bonneau ${ }^{\mathrm{d}}$ \\ a Department of Physics, Faculty of Science, Universiti Teknologi Malaysia, 81310 UTM Johor Bahru, Johor, Malaysia. \\ ${ }^{b}$ UTM Centre for Industrial and Applied Mathematics, 81310 Johor Bahru, Johor, Malaysia. \\ c Universite de Bordeaux, CENBG, UMR5797, F-33170 Gradignan, France. \\ ${ }^{d}$ CNRS, IN2P3, CENBG, UMR5797, F-33170 Gradignan, France.
}

* Corresponding author:kmhock@utm.my

\section{Article history}

Received 30 Mac 2019

Revised 14 May 2019

Accepted 5 Septembe 2019

Published Online 2 February 2020

\begin{abstract}
Nuclear structure data play an important role in nuclear physics studies and applications such as nuclear power generation. This article presents the evaluations of a mean-field approach to describe two nuclear structure quantities namely the electric and magnetic moments. The Hartree-Fock-pluspairing approach was employed with pairing correlations treated within the Bardeen-CooperSchrieffer (BCS) framework. The Skyrme SIII parametrization and seniority force were chosen to approximate the effective nucleon-nucleon and pairing interactions, respectively. Calculated results show that the self-consistent blocking procedure which considers time-reversal symmetry breaking is important to reproduce experimental magnetic moment.
\end{abstract}

Keywords: Hartree-Fock, BCS pairing, nuclear properties, time-reversal, mean-field

\section{INTRODUCTION}

Clean and renewable energy is one of the important themes in the Fourth Industrial Revolution. The aim is to change our dependency from the current fossil-fuel generated power to a more sustainable option [1]. In this aspect, nuclear fission has been a reliable source of low-carbon energy production with many countries relying on nuclear energy for electricity generation [2]. While nuclear energy is not a type of renewable energy in the strictest sense, it still plays an important role in electricity generation especially for countries that do not have access to other forms of renewable sources such as solar, hydropower, and wind. It is foreseen that nuclear energy derived from fission of a heavy nucleus into two smaller fragments will continue to be the main source of nuclear energy for many years to come.

While nuclear fission is the key element of nuclear energy generation, this process itself is not fully understood by physicists. Nuclear fission has remained as an active field of research for both nuclear theorists and experimentalists even after its first discovery in 1938 [3]. From theoretician's point of view, a unifying nuclear model capable of describing both static and dynamic nuclear properties is desired. As theoreticians strive to achieve this, results generated from theoretical studies also play an important role not only in experimental nuclear physics but also to other nuclear applications such as power generation.

Theoretical researches contribute to nuclear power generation in the form of providing information on relevant nuclear properties to be used as primary inputs for cross sections evaluations, for instance in the works $[4,5]$. One example of input parameter needed for fission cross-section calculations is the fission-barrier heights. This quantity can be extracted from the deformation energy surface describing the variation of energy with nuclear deformation. Fig. 1 shows an example of deformation energy surface together with some excited states above the saddle points obtained from liquid drop model calculations and liquid-drop-plus-shell-correction (referred to as SCM in the diagram) calculations [6].

Nowadays, many nuclear models have been developed and used to study nuclear fission process. The mean-field approach based on the Hartree-Fock-plus-pairing is one such model. This model was first developed in the 1970s to investigate nuclear properties in the groundstate deformation $[7,8]$. It was subsequently extended to large nuclear deformation and applied to the study of nuclear fission of ${ }^{240} \mathrm{Pu}$ nucleus in 1974 [9]. Recently, interest towards the application of mean-field approach in odd-mass nuclei is on the rise.

Theoretical description of odd-mass nucleus is slightly different than that encountered for even-even (even with respect to both neutron and proton numbers) nucleus. The difference is due to the unpaired nucleon in odd-mass nucleus. Two main approaches exist with this regard to treating this unpaired nucleon. It boils down to the choice of whether to account for the breaking of time-reversal symmetry at the mean-field level or not. Studies have shown that the effect of the time-reversal symmetry on total binding energy is negligible (of the order of a few eV). As such, proponents of the equal filling approximation argue that it makes sense to ignore the timereversal symmetry breaking effect altogether. On the contrary, studies on magnetic dipole moment of some odd-mass nuclei showed that proper accounting of the time-reversal symmetry breaking which results in core (of the odd-mass nucleus) polarization is crucial in reproducing qualitative agreement to experimental data [10]. Following this argument, studies have been performed within the socalled self-consistent blocking (SCB) approach for band-head 
energies of odd-mass heavy nuclei at the ground-state deformation [11] and extrapolated to larger deformation for description of fissionbarrier heights [12].

This paper extends the previous studies in actinide region into the rare-earth region as a testing ground for the SCB approach in view of rich experimental data available for comparison. Focus is given to electromagnetic moments with emphasis on magnetic dipole moment at the ground-state deformation. The mean-field approach used in this study is discussed briefly in the Theoretical Framework section with reference to key articles for in-depth information. The numerical methods necessary to solve the differential equation are mentioned in Technical Details of Calculations section. Results are presented in the following section and the conclusion from this study is drawn in the last section.

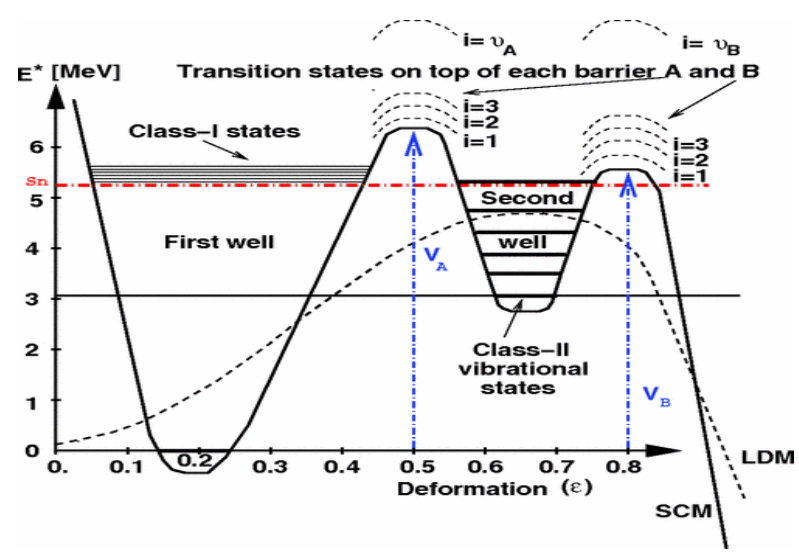

Fig. 1 Deformation energy surfaces obtained from liquid drop model (LDM) and self-correction method (SCM). The inner and outer fissionbarrier heights are denoted as $\mathrm{V}_{\mathrm{A}}$ and $\mathrm{V}_{\mathrm{B}}$, respectively. The diagram was extracted from Bouland et al. [6] with permission from American Physical Society.

\section{THEORETICAL FRAMEWORK}

\section{The Hartree-Fock-plus-BCS approach}

When solving the Hamiltonian for a many-particle system such as in atomic nucleus, one can rely on the Hartree-Fock approximation to reduce from a many-particle to a simplified one-particle problem. The many-particle Hamiltonian $\widehat{H}$ is written as

$$
\widehat{H}=\widehat{K}+\widehat{V},
$$

where $\widehat{K}$ is the total kinetic energy and $\widehat{V}$ consists of two (sometimes three) -particle term. In nuclear physics, the latter is the nucleonnucleon interaction and is usually represented by a phenomenological mathematical expression. The Skyrme interaction is one such example and has been widely used in nuclear structure [13] and reaction [14] studies.

When using the Skyrme interaction, the expectation value of the many-particle Hamiltonian in a normalized Slater determinant $|\Psi\rangle$ can be written in terms of a Hamiltonian density $\widehat{\mathcal{H}}(\boldsymbol{r})$

$$
\langle\Psi|\widehat{H}| \Psi\rangle=\int \widehat{\mathcal{H}}(\boldsymbol{r}) d r
$$

with contributions to the Hamiltonian density coming from the kinetic energy $\widehat{\mathcal{H}}_{\text {kin }}$, central $\widehat{\mathcal{H}}_{c}$, density-dependent $\widehat{\mathcal{H}}_{D D}$, spin-orbit $\widehat{\mathcal{H}}_{\text {s.o }}$, and Coulomb energy $\widehat{\mathcal{H}}_{\text {Coul }}$ terms

$$
\widehat{\mathcal{H}}(\boldsymbol{r})=\widehat{\mathcal{H}}_{\text {kin }}(\boldsymbol{r})+\widehat{\mathcal{H}}_{c}(\boldsymbol{r})+\widehat{\mathcal{H}}_{D D}(\boldsymbol{r})+\widehat{\mathcal{H}}_{\text {s.o }}(\boldsymbol{r})+\widehat{\mathcal{H}}_{\text {Coul }}(\boldsymbol{r}) .
$$

The contributions to the Hamiltonian density are written in terms of six types of local densities that are classified as either time-even or time-odd local densities depending on the action of the time-reversal symmetry operator.

The Hartree-Fock method is an approximation approach to solve the many-particle problem in terms of a one-particle problem. The one-particle Hartree-Fock Hamiltonian is obtained here by varying the expectation value of $\widehat{H}$ (i.e. the total energy) with respect to $|\Psi\rangle$. In practice, this is equivalent to making a variation with respect to the single-particle wave function $\phi_{i}(\boldsymbol{r})$ since the Slater determinant for $\mathrm{N}$-particle system is written as

$$
\langle\boldsymbol{r} \mid \Psi\rangle=\Psi(\boldsymbol{r})=\frac{1}{\sqrt{N !}}\left|\begin{array}{ccc}
\phi_{1}\left(\boldsymbol{r}_{1}\right) & \cdots & \phi_{1}\left(\boldsymbol{r}_{N}\right) \\
\vdots & \ddots & \vdots \\
\phi_{N}\left(\boldsymbol{r}_{1}\right) & \cdots & \phi_{N}\left(\boldsymbol{r}_{N}\right)
\end{array}\right| .
$$

Applying the variational method yields a set of Hartree-Fock equations to be solved iteratively (see Appendix A of [12] and references therein). One usually starts by using previously converged Hartree-Fock solution as input, which is then used to compute the local densities. The local densities are then used for solving the Hartree-Fock equations yielding a new set of wave functions. The wave functions are then used to calculate local densities and the calculations are repeated until convergence is achieved.

When describing non-closed shell nucleus, it is important to consider pairing correlations. Within the HF framework, pairing correlations can be treated using the Bardeen-Cooper-Schrieffer (BCS) [15] approach. In doing this, one enters the BCS loop immediately before calculations of local densities in the HF iterative process. The occupancy probability $v_{i}^{2}$ of each single-particle wave function is calculated resulting in the inclusion of $v_{i}^{2}$ term in the local densities. The value of $v_{i}^{2}$ can take any value between 0 to 1 with $v_{i}^{2}=1$ representing that the single-particle state $i$ is fully occupied.

\section{Approach to odd-mass nucleus}

For calculations of odd-mass nucleus, we specify at the start of the calculation which single-particle state that will be occupied by the last unpaired nucleon. The projection of the total angular momentum on the symmetry $z$-axis of the blocked $k$ state, $\Omega_{k}$ is assumed to be the theoretical total angular momentum quantum number of the whole nucleus denoted as $K$. Several calculations were performed by blocking different single-particle states in order to determine the lowest-energy solution for one particular nucleus.

The blocking calculation herein is referred to as SCB approach in $[11,12]$ and is performed by setting the occupation probability of the specific single-particle state to 1 . Calculations are performed similar to an even-even nucleus with the exception of the blocked state which does not participate in the BCS calculations. The blocking procedure causes the time-odd local densities to be non-vanishing and induces the breaking of time reversal symmetry in the HF level. This results in a single-particle spectrum which is no longer degenerate as in the case for the ground-state of an even-even nucleus.

Consequently, one is no longer able to establish a one-to-one correspondence between the single-particle states necessary to define a BCS-pair state. A method initially applied in [16] was used to reestablish this pair-state by searching for the maximum overlap between one single-particle state and another time-conjugate state.

\section{TECHNICAL DETAILS OF CALCULATIONS}

\section{Choice of interactions}

The Skyrme interaction is written in terms of some parameters have been optimized according to specific study purpose. In this work, the SIII Skyrme parametrization [17] was chosen in view of its suitability for the study of ground-state nuclear properties. The values of the Skyrme parameters for the SIII set can be retrieved as presented in Appendix A of [18].

Within the BCS framework, the seniority force has been chosen to approximate residual pairing interaction. The pairing matrix element is given by

$$
v_{k \hat{k} l \hat{l}}=-\frac{G_{q}}{11+N_{q}},
$$

where $G_{q}$ is the pairing strength and $N_{q}$ is the nucleon number for the charge state $q$. A systematic study on the fit procedure of the pairing 
strengths has been performed in [19] and the optimal values around the rare earth region are found to be $G_{n}=16 \mathrm{MeV}$ (for neutron) and

$$
G_{p}=15 \mathrm{MeV} \text { (for proton) }
$$

\section{Numerical integration}

The single-particle wave function $\phi_{i}$ in our approach is expanded on the deformed harmonic oscillator basis and is written in terms of the Hermite polynomial $H_{n}(\xi)$ along the symmetry $z$-axis and Laguerre polynomial $L_{n}(\eta)$ at the perpendicular $r$-plane with

$$
\xi=z \beta_{z} \quad \eta=r^{2} \beta_{\perp}^{2}
$$

in which $\beta_{z}$ and $\beta_{\perp}$ are harmonic oscillator constants. The numerical integration involving the HF Hamiltonian is solved by using the Gauss-Hermite and Gauss-Laguerre quadrature methods such that

$$
\iint e^{-\xi^{2}} e^{-\eta} g(\xi, \eta) d \xi d \eta \approx \sum_{i=1}^{N_{G}^{(z)}} \sum_{j=1}^{N_{G}^{(r)}} \omega_{i} \omega_{j} f\left(\xi_{i}\right) f\left(\eta_{j}\right),
$$

where $\omega_{i}$ and $\omega_{j}$ are respective weighting factors [20]

$$
\begin{aligned}
& \omega_{i}=\frac{2^{\left(N_{G}^{(z)}-1\right)} N_{G}^{(z)} ! \sqrt{\pi}}{N_{G}^{(z)}{ }^{2}\left(H_{N_{G}^{(z)}-1}\left(\xi_{i}\right)\right)^{2}}, \\
& \omega_{j}=\frac{\eta_{j}}{\left(N_{G}^{(r)}+1\right)^{2}\left(L_{N_{G}^{(r)}+1}\left(\eta_{j}\right)\right)^{2}} .
\end{aligned}
$$

The number of Gauss-Hermite point $N_{G}^{(z)}$ and Gauss-Laguerre point $N_{G}^{(r)}$ are chosen here to be 50 and 16 , respectively.

\section{RESULTS AND DISCUSSION}

\section{Nuclear spin and parity}

As mentioned in the Theoretical Framework section, calculations for an odd-mass nucleus involved computations of different blocked single-particle states. The theoretical ground-state solution corresponds to the lowest-energy solution and the total angular momentum $K$ and parity $\pi$ for the whole nucleus is approximated by the $\Omega_{K}$ and $\pi$ quantum numbers of the blocked single-particle state.

The ground-state $K^{\pi}$ quantum numbers of some odd-neutron nuclei (i.e. nuclei with odd numbers of neutrons but even numbers of protons) are tabulated in column 2 of Table 1.
The experimental nuclear spin and parity $I^{\pi}$ quantum numbers are reproduced from our calculations except for ${ }^{165} \mathrm{Dy},{ }^{169} \mathrm{Er}$, and ${ }^{171} \mathrm{Yb}$. For these three nuclei, the energy difference between the two blocked states of the same nucleus is about $100 \mathrm{keV}$.

\section{Magnetic dipole moment}

The magnetic dipole moment $\mu_{\text {tot }}$ of a nucleus is a sum of contribution from intrinsic $\mu_{\text {intr }}$ and collective $\mu_{\text {coll }}$ magnetic moments

$$
\mu_{\text {tot }}=\mu_{\text {intr }}+\mu_{\text {coll }} .
$$

Calculations for both contributing terms were performed according to equations (6-8) and (12-13) of [10]. The calculated values are tabulated in column 3 and compared to experimental data [21] in column 4 of Table 1 . The results show that the calculated values agree well with experimental data except for three nuclei namely ${ }^{171} \mathrm{Er}$ and the $7 / 2^{+}$blocked configuration in both ${ }^{169} \mathrm{Er}$ and ${ }^{171} \mathrm{Yb}$. In these nuclei, the magnitude of the calculated results and experimental values are very close except that they are opposite in sign. When excluding these major differences, the largest discrepancy between calculated and experimental $\mu_{\text {tot }}$ is about $0.4 \mu_{N}$ in ${ }^{175} \mathrm{Yb}$ nucleus.

\section{Electric quadrupole moment}

The electric charge quadrupole moment which provides information on charge distribution is calculated within the HF-BCS framework using the equation [22]

$$
Q_{20}=\int \rho^{(p)}(\boldsymbol{r})\left(3 z^{2}-r^{2}\right) d^{3} \boldsymbol{r}
$$

with $r^{2}=x^{2}+y^{2}$ and $\rho^{(p)}(\boldsymbol{r})$ is the proton charge density. The intrinsic charge quadrupole moment $Q_{20}$ provides the charge distribution in the frame of reference where the nucleus is at rest. Comparison to experimental data is made using the spectroscopic quadrupole moment $Q_{20}^{(s)}$ obtained from the expression [23]

$$
Q_{20}^{(s)}=\frac{3 K^{2}-I(I+1)}{(I+1)(2 I+3)} Q_{20},
$$

where $I$ is the experimental nuclear spin quantum number. The

\begin{tabular}{|c|c|c|c|c|c|}
\hline \multirow[t]{2}{*}{ Nucleus } & \multirow[t]{2}{*}{$K^{\pi}$} & \multicolumn{2}{|c|}{$\mu_{t o t}\left[\mu_{N}\right]$} & \multicolumn{2}{|c|}{$Q_{20}^{(s)}[b]$} \\
\hline & & Theo & Exp & Theo & Exp \\
\hline${ }^{163} \mathrm{Dy}$ & $5 / 2$ & 0.885 & $0.673(4)$ & 2.701 & $\begin{array}{c}\text { Z.Jाणण } \\
2.65(2)\end{array}$ \\
\hline${ }^{165} \mathrm{Dy}$ & $\begin{array}{l}7 / 2^{+}(\exp ) \\
1 / 2^{-} \text {(theo) }\end{array}$ & $\begin{array}{c}-0.469 \\
0.427\end{array}$ & $\begin{array}{c}-0.520(5) \\
-\end{array}$ & $\begin{array}{c}3.586 \\
-\end{array}$ & $\begin{array}{c}\text { J.4071 } \\
-\end{array}$ \\
\hline${ }^{169} \mathrm{Er}$ & $\begin{array}{l}1 / 2^{-}(\exp ) \\
7 / 2^{+} \text {(theo) }\end{array}$ & $\begin{array}{c}0.409 \\
-0.493 \\
\end{array}$ & $\begin{array}{c}0.52(3) \\
0.4850(2)\end{array}$ & $\begin{array}{c}- \\
3.686 \\
\end{array}$ & - \\
\hline${ }^{171} \mathrm{Er}$ & $5 / 2$ & -0.695 & $0.659(10)$ & 2.801 & $2.86(9)$ \\
\hline${ }^{171} \mathrm{Yb}$ & $\begin{array}{l}1 / 2^{-}(\exp ) \\
7 / 2^{+} \text {(theo) } \\
\end{array}$ & $\begin{array}{c}0.404 \\
-0.480 \\
\end{array}$ & $\begin{array}{c}0.49367(1) \\
0.4949(4) \\
\end{array}$ & $\begin{array}{c}- \\
3.725 \\
\end{array}$ & - \\
\hline${ }^{173} \mathrm{Yb}$ & $5 / 2$ & -0.686 & $\begin{array}{c}-0.648(3) \\
-0.67989(3) \\
0.68002(3) \\
\end{array}$ & 2.823 & $2.8(4)$ \\
\hline${ }^{175} \mathrm{Yb}$ & $7 / 2$ & 1.179 & $\begin{array}{c}0.768(8) \\
0.58(8) \\
0.40(5) \\
\end{array}$ & 3.588 & $3.52(5)$ \\
\hline${ }^{177} \mathrm{Hf}$ & $7 / 2$ & 1.067 & 0.7935 & 3.499 & $3.36(3)$ \\
\hline${ }^{179} \mathrm{Hf}$ & $9 / 2^{+}$ & -0.653 & $-0.6409(13)$ & 3.928 & 3.1901 \\
\hline
\end{tabular}
calculated $Q_{20}^{(s)}$ are tabulated and compared to experimental data $[21,24]$ in columns 5 and 6 of Table 1, respectively. Excluding ${ }^{169} \mathrm{Er}$ and ${ }^{171} \mathrm{Yb}$ in which no experimental data is available for comparison, it was found that the calculated values lie within $\pm 0.15 \mathrm{~b}$ from experimental values.

Table 1 Magnetic dipole moment tot (in unit of nuclear magneton $\mu_{N}$ ) and electric charge quadrupole moment $Q_{20}$ (in unit of barns, $b$ ) for oddneutron nuclei with comparison to experimental data extracted from [21, 24]. The values in parentheses reflect uncertainties in the last digits. 


\section{CONCLUSION}

In this paper, the SCB approach to odd-mass nucleus within the HF-BCS approach has been tested for some rare earth nuclei. Based on the small samples of nuclei and choice of nuclear properties presented herein, comparison between theoretical and experimental data show that the SCB approach is a reliable manner to treat oddmass nucleus. A remarkable point is the success in reproducing some experimental magnetic moment which is a quantity where the effect of time-reversal symmetry breaking is the most apparent. Nevertheless, some large deviations from data were found and this points toward some improvements to be considered for the next stage of our work.

\section{ACKNOWLEDGEMENT}

Meng-Hock Koh would like to acknowledge Universiti Teknologi Malaysia (UTM) for the financial support provided which allows the presentation of this work in the ISMI-ICTAS 2018 conference through the grant number Q.J130000.2626.15J74. Next, Nurhafiza M. Nor and Nor-Anita Rezle would like to show their appreciation to UTM for supporting their postgraduate studies through the same research grant.

\section{REFERENCES}

[1] PwC UK, Harnessing the fourth industrial revolution for sustainable emerging cities. Fourth industrial revolution for the earth series. [online] Available at https://www.pwc.com/gx/en/sustainability/assets/ 4ir-forthe-earth.pdf [Accessed 11 July 2018].

[2] U.S. Department of Energy, Available at https://www.eia.gov/energy explained/index.php?page=nuclear_power_plants. [Accessed 11 July 2018].

[3] Meitner, L. and Frisch, O. R. Disintegration of uranium by neutrons: A new type of nuclear reaction. Nature. 1939. 143: 239-240.

[4] Goriely, S., Hilaire, S., Koning, A. J., Sin, M., Capote, R. Towards a prediction of fission cross sections on the basis of microscopic nuclear inputs. Phys. Rev. C. 2009. 79: 024612.

[5] Capote, R., Herman, M., Oblozinsky, P., Young, P-G., Goriely, S., Belgya, T., Ignatyuk, A.-V., Koning, A.-J., Hilaire, S., Plujko, V.-A., Avrigeanu, M., Bersillon, O., Chadwick, M.-B., Fukahori, T., Ge, Z., Han, Y., Kailas, S., Kopecky, J., Maslov, V.-M., Reffo, G., Sin, M., Soukhovitskii, E.-Sh., Talou, P. RIPL - Reference Input Parameter Library for calculation of nuclear reactions and nuclear data evaluations. Nucl. Data Sheets. 2009. 110: 3107.

[6] Bouland, O., Lynn, J. E. and Talou, P. R-matrix analysis and prediction of low-energy neutron-induced fission cross sections for a range of $\mathrm{Pu}$ isotopes. Phys. Rev. C. 2013.88: 054612.
[7] Vautherin, D., Brink, D. M. Hartree-Fock calculations with Skyrmes interaction. I. Spherical nuclei. Phys. Rev. C. 1972. 5: 626-647.

[8] Vautherin, D. Hartree-Fock calculations with Skyrmes interaction. II. Axially deformed nuclei. Phys. Rev. C. 1973. 7: 296-316.

[9] Flocard, H., Quentin, P., Vautherin, D., Veneroni, M. and Kerman, A. K. Self-consistent calculation of the fission barrier of ${ }^{240} \mathrm{Pu}$. Nucl. Phys. A. 1974. 231: 176.

[10] Bonneau, L., Minkov, N., Duc, D. D., Quentin, P. and Bartel, J. Effect of core polarization on magnetic dipole moments in deformed odd-mass nuclei. Phys. Rev. C. 2015. 91: 054307.

[11] Koh, M.-H., Duc, D. D., Hao, T. V. N., Ha, T. L., Quentin, P., Bonneau, L. Band-head spectra of low-energy single-particle excitations in some well-deformed, odd-mass heavy nuclei within a microscopic approach. Eur. Phys. J. A. 2016. 52: 3.

[12] Koh, M.-H., Bonneau, L., Quentin, P., Nhan Hao, T. V., Wagiran, H. Fission barriers of two odd-neutron actinide nuclei taking into account the time-reversal symmetry breaking at the mean-field level. Phys. Rev. C. 2017. 95: 014315.

[13] Bender, M., Heenen, P.-H. and Reinhard, P.-G. Self-consistent meanfield models for nuclear structure. Rev. Mod. Phys. 2003. 75: 121-180.

[14] Nobre, G. P. A., Dietrich, F. S., Escher, J. E., Thompson, I. J., Dupuis, M., Terasaki, J. and Engel, J. Toward a microscopic reaction description based on energy-density-functional structure models. Phys. Rev. C. 2011. 84: 064609.

[15] Bardeen, J., Cooper, L. N., and Schrieffer, J. R. Theory of superconductivity. Phys.Rev. 1957. 108: 1175-1204.

[16] Pototzky, K. J., Erler, J., Reinhard, P.-G., Nesterenko, V.O. Properties of odd nuclei and the impact of time-odd mean fields: A systematic Skyrme-Hartree-Fock analysis. Eur. Phys. J. A. 2010. 46: 299-313.

[17] Beiner, M., Flocard, H., Giai, N. V., Quentin, P. Nuclear ground-state properties and self-consistent calculations with the Skyrme interaction. (I). Spherical Description Nucl. Phys. A. 1975. 238: 29-69.

[18] Koh, M.-H. Fission-barriers and energy spectra of odd-mass actinide nuclei in self-consistent mean-eld calculations. $\mathrm{PhD}$. thesis. University of Bordeaux - Universiti Teknologi Malaysia. 2015.

[19] Nor, N. M., Rezle, N.-A., Kelvin-Lee, K.-W., Koh, M.-H., Bonneau, L., Quentin, P. Consistency of two different approaches to determine the strength of a pairing residual interaction in the rare-earth region. Phys. Rev. C. 2019. 99: 064306.

[20] Abramowitz, M. and Stegun, I. A. Handbook of mathematical functions Dover. New York. 1968, p. 890.

[21] Stone, N. J. Table of nuclear magnetic dipole and electric quadrupole moments. At. Data Nucl. Data Tables 2005. 90: 75-176.

[22] K. Krane, Introductory Nuclear Physics, John Wiley and Sons, United States of America, 1988, p. 74.

[23] A. Bohr, B.R. Mottelson, Nuclear Structure, Vol. 2. Benjamin, Reading, 1975 , p. 45.

[24] Stone, N. J. Table of nuclear electric quadrupole moments At. Data Nucl. Data Tables. 2016. 1: 111-112. 